\title{
Highly Efficient Dye-sensitized Solar Cells with Composited Food Dyes
}

Dafu Wang, Wei Wei, and Yun Hang Hu*

Department of Materials Science and Engineering, Michigan Technological University, 1400

Townsend Drive, Houghton, Michigan, USA, 49931 *E-mail: yunhangh@mtu.edu 
Table S1. Efficiency, fill factor, short circuit current, and open circuit voltage of single-dye solution of Amaranth with $\mathrm{ZnO} / \mathrm{FTO}$ photoelectrodes.

\begin{tabular}{lllll}
\hline Concentration & Efficiency & Fill factor & Short circuit current & Open circuit voltage \\
$(\mathrm{mmol} / \mathrm{L})$ & $(\%)$ & & $\left(\mathrm{mA} \mathrm{cm}^{-2}\right)$ & $(\mathrm{V})$ \\
\hline 0 & 0.02 & 0.27 & 0.06 & 0.59 \\
0.1 & 0.18 & 0.11 & 1.45 & 0.43 \\
0.2 & 0.15 & 0.15 & 0.87 & 0.46 \\
0.3 & 0.23 & 0.15 & 1.43 & 0.43 \\
0.6 & 0.18 & 0.16 & 0.89 & 0.49 \\
\hline
\end{tabular}


Table S2. Efficiency, fill factor, short circuit current, and open circuit voltage of single-dye solution of Erythrosin B with $\mathrm{ZnO} / \mathrm{FTO}$ photoelectrodes.

\begin{tabular}{lllll}
\hline Concentration & Efficiency & Fill factor & Short circuit current & Open circuit voltage \\
$(\mathrm{mmol} / \mathrm{L})$ & $(\%)$ & & $\left(\mathrm{mA} \mathrm{cm}^{-2}\right)$ & $(\mathrm{V})$ \\
\hline 0 & 0.02 & 0.27 & 0.06 & 0.59 \\
0.1 & 0.43 & 0.26 & 1.18 & 0.56 \\
0.2 & 0.64 & 0.23 & 2.07 & 0.54 \\
0.3 & 0.92 & 0.23 & 3.26 & 0.50 \\
0.6 & 0.90 & 0.22 & 3.22 & 0.51 \\
\hline
\end{tabular}


Table S3. Efficiency, fill factor, short circuit current, and open circuit voltage of single-dye solution of Sunset Yellow with ZnO/FTO photoelectrodes.

\begin{tabular}{lllll}
\hline Concentration & Efficiency & Fill factor & Short circuit current & Open circuit voltage \\
$(\mathrm{mmol} / \mathrm{L})$ & $(\%)$ & & $\left(\mathrm{mA} \mathrm{cm}^{-2}\right)$ & $(\mathrm{V})$ \\
\hline 0 & 0.02 & 0.27 & 0.06 & 0.59 \\
0.1 & 0.65 & 0.33 & 1.67 & 0.47 \\
0.2 & 0.49 & 0.20 & 2.32 & 0.42 \\
0.3 & 0.23 & 0.20 & 1.14 & 0.40 \\
0.6 & 0.21 & 0.17 & 1.24 & 0.40 \\
\hline
\end{tabular}


Table S4. Efficiency, fill factor, short circuit current, and open circuit voltage of single-dye solution of Amaranth with $\mathrm{TiO}_{2} / \mathrm{FTO}$ photoelectrodes.

\begin{tabular}{lllll}
\hline Concentration & Efficiency & Fill factor & Short circuit current & Open circuit voltage \\
$(\mathrm{mmol} / \mathrm{L})$ & $(\%)$ & & $\left(\mathrm{mA} \mathrm{cm}^{-2}\right)$ & $(\mathrm{V})$ \\
\hline 0 & 0.02 & 0.31 & 0.06 & 0.51 \\
0.1 & 0.08 & 0.15 & 1.23 & 0.17 \\
0.2 & 0.14 & 0.16 & 1.08 & 0.33 \\
0.3 & 0.15 & 0.16 & 1.06 & 0.36 \\
0.6 & 0.17 & 0.24 & 0.07 & 0.40 \\
\hline
\end{tabular}


Table S5. Efficiency, fill factor, short circuit current, and open circuit voltage of single-dye solution of Erythrosin B with $\mathrm{TiO}_{2} / \mathrm{FTO}$ photoelectrodes.

\begin{tabular}{lllll}
\hline Concentration & Efficiency & Fill factor & Short circuit current & Open circuit voltage \\
$(\mathrm{mmol} / \mathrm{L})$ & $(\%)$ & & $\left(\mathrm{mA} \mathrm{cm}^{-2}\right)$ & $(\mathrm{V})$ \\
\hline 0 & 0.02 & 0.31 & 0.06 & 0.51 \\
0.1 & 0.27 & 0.13 & 2.12 & 0.40 \\
0.2 & 0.26 & 0.12 & 2.10 & 0.40 \\
0.3 & 0.62 & 0.23 & 1.96 & 0.55 \\
0.6 & 0.17 & 0.08 & 1.94 & 0.44 \\
\hline
\end{tabular}


Table S6. Efficiency, fill factor, short circuit current, and open circuit voltage of single-dye solution of Sunset Yellow with $\mathrm{TiO}_{2} / \mathrm{FTO}$ photoelectrodes.

\begin{tabular}{lllll}
\hline Concentration & Efficiency & Fill factor & Short circuit current & Open circuit voltage \\
$(\mathrm{mmol} / \mathrm{L})$ & $(\%)$ & & $\left(\mathrm{mA} \mathrm{cm}^{-2}\right)$ & $(\mathrm{V})$ \\
\hline 0 & 0.02 & 0.31 & 0.06 & 0.51 \\
0.1 & 0.48 & 0.23 & 2.02 & 0.42 \\
0.2 & 0.49 & 0.31 & 1.52 & 0.41 \\
0.3 & 0.13 & 0.11 & 1.32 & 0.34 \\
0.6 & 0.17 & 0.16 & 1.22 & 0.35 \\
\hline
\end{tabular}


Table S7. Efficiency, fill factor, short circuit current, and open circuit voltage of combined-dye solution of Sunset Yellow (SY) and Erythrosin B (EB), in different ratio of combination, with $\mathrm{ZnO/FTO} \mathrm{photoelectrodes.}$

\begin{tabular}{|c|c|c|c|c|}
\hline Ratio & Efficiency & Fill & Short circuit current & Open circuit voltage \\
\hline (SY\%/EB\%) & $(\%)$ & factor & $\left(\mathrm{mA} \mathrm{cm}{ }^{-2}\right)$ & (V) \\
\hline $0 / 100$ & 0.92 & 0.36 & 2.07 & 0.51 \\
\hline $20 / 80$ & 0.38 & 0.18 & 2.22 & 0.39 \\
\hline $30 / 70$ & 0.89 & 0.21 & 4.24 & 0.40 \\
\hline $40 / 60$ & 1.40 & 0.20 & 6.24 & 0.46 \\
\hline $50 / 50$ & 1.64 & 0.05 & 19.28 & 0.64 \\
\hline $60 / 40$ & 1.29 & 0.04 & 20.72 & 0.65 \\
\hline $70 / 30$ & 0.35 & 0.02 & 21.28 & 0.41 \\
\hline $80 / 20$ & 0.29 & 0.02 & 16.04 & 0.49 \\
\hline $100 / 0$ & 0.23 & 0.10 & 2.32 & 0.41 \\
\hline
\end{tabular}


Table S8. Efficiency, fill factor, short circuit current, and open circuit voltage of combined-dye solution of Sunset Yellow (SY) and Erythrosin B (EB), in different ratio of combination, with $\mathrm{TiO}_{2} /$ FTO photoelectrodes.

\begin{tabular}{|c|c|c|c|c|}
\hline Ratio & Efficiency & Fill & Short circuit current & Open circuit voltage \\
\hline (SY\%/EB\%) & $(\%)$ & factor & $\left(\mathrm{mA} \mathrm{cm}{ }^{-2}\right)$ & (V) \\
\hline $0 / 100$ & 0.62 & 0.21 & 2.10 & 0.55 \\
\hline $20 / 80$ & 0.25 & 0.15 & 1.80 & 0.37 \\
\hline $30 / 70$ & 0.34 & 0.16 & 1.88 & 0.46 \\
\hline $40 / 60$ & 0.38 & 0.15 & 1.89 & 0.56 \\
\hline $50 / 50$ & 1.54 & 0.24 & 3.99 & 0.63 \\
\hline $60 / 40$ & 0.38 & 0.17 & 2.22 & 0.41 \\
\hline $70 / 30$ & 0.38 & 0.17 & 2.18 & 0.40 \\
\hline $80 / 20$ & 0.37 & 0.18 & 2.15 & 0.38 \\
\hline $100 / 0$ & 0.13 & 0.10 & 1.52 & 0.34 \\
\hline
\end{tabular}


Table S9. Efficiency, fill factor, short circuit current, and open circuit voltage of combined-dye solution of Amaranth (AM) and Erythrosin B (EB), in different ratio of combination, with $\mathrm{ZnO} / \mathrm{FTO}$ photoelectrodes.

\begin{tabular}{|c|c|c|c|c|}
\hline $\begin{array}{l}\text { Ratio } \\
(\mathrm{AM} \% / \mathrm{EB} \%)\end{array}$ & $\begin{array}{l}\text { Efficiency } \\
(\%)\end{array}$ & $\begin{array}{l}\text { Fill } \\
\text { factor }\end{array}$ & $\begin{array}{l}\text { Short circuit current } \\
(\mathrm{mA} \mathrm{cm}-2)\end{array}$ & $\begin{array}{l}\text { Open circuit voltage } \\
\text { (V) }\end{array}$ \\
\hline $0 / 100$ & 0.92 & 0.36 & 3.26 & 0.49 \\
\hline $20 / 80$ & 0.93 & 0.17 & 5.24 & 0.42 \\
\hline $30 / 70$ & 1.48 & 0.20 & 7.04 & 0.43 \\
\hline $40 / 60$ & 2.16 & 0.20 & 8.40 & 0.53 \\
\hline $50 / 50$ & 2.46 & 0.23 & 7.84 & 0.54 \\
\hline $60 / 40$ & 2.64 & 0.21 & 10.36 & 0.52 \\
\hline $70 / 30$ & 1.55 & 0.22 & 5.92 & 0.47 \\
\hline $80 / 20$ & 0.93 & 0.15 & 5.52 & 0.44 \\
\hline $100 / 0$ & 0.23 & 0.25 & 1.78 & 0.44 \\
\hline
\end{tabular}


Table S10. Efficiency, fill factor, short circuit current, and open circuit voltage of combined-dye solution of Amaranth (AM) and Erythrosin B (EB), in different ratio of combination, with $\mathrm{TiO}_{2} /$ FTO photoelectrodes.

\begin{tabular}{|c|c|c|c|c|}
\hline $\begin{array}{l}\text { Ratio } \\
\text { (AM\%/EB\%) }\end{array}$ & $\begin{array}{l}\text { Efficiency } \\
(\%)\end{array}$ & $\begin{array}{l}\text { Fill } \\
\text { factor }\end{array}$ & $\begin{array}{l}\text { Short circuit current } \\
\left.(\mathrm{mA} \mathrm{cm})^{-2}\right)\end{array}$ & $\begin{array}{l}\text { Open circuit voltage } \\
\text { (V) }\end{array}$ \\
\hline $0 / 100$ & 0.62 & 0.21 & 2.10 & 0.55 \\
\hline $20 / 80$ & 1.24 & 0.19 & 6.08 & 0.43 \\
\hline $30 / 70$ & 1.31 & 0.20 & 5.80 & 0.45 \\
\hline $40 / 60$ & 1.36 & 0.19 & 6.20 & 0.46 \\
\hline $50 / 50$ & 1.41 & 0.20 & 6.24 & 0.46 \\
\hline $60 / 40$ & 1.27 & 0.18 & 5.68 & 0.50 \\
\hline $70 / 30$ & 0.89 & 0.12 & 5.88 & 0.53 \\
\hline $80 / 20$ & 0.38 & 0.14 & 2.77 & 0.41 \\
\hline $100 / 0$ & 0.15 & 0.15 & 1.08 & 0.36 \\
\hline
\end{tabular}


Table S11. Efficiency, fill factor, short circuit current, and open circuit voltage of combined-dye solution of Amaranth (AM) and Sunset Yellow (SY), in different ratio of combination, with ZnO/FTO photoelectrodes.

\begin{tabular}{|c|c|c|c|c|}
\hline $\begin{array}{l}\text { Ratio } \\
\text { (AM\%/SY\%) }\end{array}$ & $\begin{array}{l}\text { Efficiency } \\
(\%)\end{array}$ & $\begin{array}{l}\text { Fill } \\
\text { factor }\end{array}$ & $\begin{array}{l}\text { Short circuit current } \\
(\mathrm{mA} \mathrm{cm}-2)\end{array}$ & $\begin{array}{l}\text { Open circuit voltage } \\
\text { (V) }\end{array}$ \\
\hline $0 / 100$ & 0.23 & 0.10 & 2.32 & 0.41 \\
\hline $20 / 80$ & 0.43 & 0.15 & 2.37 & 0.50 \\
\hline $30 / 70$ & 0.69 & 0.09 & 8.12 & 0.42 \\
\hline $40 / 60$ & 1.04 & 0.18 & 5.72 & 0.41 \\
\hline $50 / 50$ & 1.38 & 0.19 & 6.36 & 0.46 \\
\hline $60 / 40$ & 1.40 & 0.19 & 6.24 & 0.47 \\
\hline $70 / 30$ & 1.13 & 0.18 & 5.92 & 0.47 \\
\hline $80 / 20$ & 0.50 & 0.21 & 1.85 & 0.52 \\
\hline $100 / 0$ & 0.23 & 0.25 & 0.87 & 0.43 \\
\hline
\end{tabular}


Table S12. Efficiency, fill factor, short circuit current, and open circuit voltage of combined-dye solution of Amaranth (AM) and Sunset Yellow (SY), in different ratio of combination, with $\mathrm{TiO}_{2} /$ FTO photoelectrodes.

\begin{tabular}{|c|c|c|c|c|}
\hline $\begin{array}{l}\text { Ratio } \\
\text { (AM\%/SY\%) }\end{array}$ & $\begin{array}{l}\text { Efficiency } \\
(\%)\end{array}$ & $\begin{array}{l}\text { Fill } \\
\text { factor }\end{array}$ & $\begin{array}{l}\text { Short circuit current } \\
\left.(\mathrm{mA} \mathrm{cm})^{-2}\right)\end{array}$ & $\begin{array}{l}\text { Open circuit voltage } \\
\text { (V) }\end{array}$ \\
\hline $0 / 100$ & 0.13 & 0.10 & 1.52 & 0.34 \\
\hline $20 / 80$ & 0.32 & 0.17 & 1.73 & 0.44 \\
\hline $30 / 70$ & 0.34 & 0.18 & 1.66 & 0.47 \\
\hline $40 / 60$ & 0.40 & 0.18 & 1.91 & 0.48 \\
\hline $50 / 50$ & 0.40 & 0.22 & 1.63 & 0.46 \\
\hline $60 / 40$ & 0.39 & 0.18 & 1.86 & 0.47 \\
\hline $70 / 30$ & 0.32 & 0.16 & 1.53 & 0.52 \\
\hline $80 / 20$ & 0.30 & 0.18 & 1.58 & 0.43 \\
\hline $100 / 0$ & 0.15 & 0.15 & 1.08 & 0.36 \\
\hline
\end{tabular}


Table S13. Results of $\mathrm{I}_{\mathrm{sc}}$, current density, and the Max Jsc with "100\%" EQE of the incident photon-to-electron conversion efficiency test for $\mathrm{ZnO} / \mathrm{FTO}$ photoelectrodes samples with different combination ratio between Amaranth and Erythrosin $\mathrm{B}(\mathrm{ZnO}$ electrode, $\mathrm{C}=0.3$ $\mathrm{mmol} / \mathrm{L})$.

\begin{tabular}{llll}
\hline Composition Ratio & $\mathrm{I}_{\mathrm{sc}}-\mathrm{IV}$ & $\mathrm{I}_{\mathrm{sc}}-\mathrm{IPCE}$ & $\begin{array}{l}\text { Max } \mathrm{I}_{\mathrm{sc}} \text { with "100\%" EQE } \\
\left(\mathrm{mA} \mathrm{cm}^{-2}\right)\end{array}$ \\
$\mathrm{AM} \% / \mathrm{EB} \%$ & $\left(\mathrm{~mA} \mathrm{~cm}{ }^{-2}\right)$ & $\left.(\mathrm{mA} \mathrm{cm})^{-2}\right)$ & 1.03 \\
\hline $0 / 100$ & 0.32 & 0.35 & 1.18 \\
$60 / 40$ & 0.33 & 0.37 & 0.88 \\
$100 / 0$ & 0.15 & 0.23 & \\
\hline
\end{tabular}

